\title{
Case Study of Using Life Cycle Impact Assessment in Environmental Impact Assessment
}

\author{
Kevin Fong-Rey Liu, Si-Yu Chiu, Po-Chung Yeh, and Jong-Yih Kuo
}

\begin{abstract}
Environmental impact assessment (EIA) and strategic environmental assessment (SEA) are procedural tools for environmental management that identify, predict, evaluate and mitigate the environmental impact of development proposals or policies. Life cycle impact assessment (LCIA) is a common analytical tool for environmental management. The use of the LCIA for the preparation of EIA and SEA reports clearly shows the causal linkage for hazard-pathway-receptor-damage and better determines the significance of the impact. Firstly, the use of the LCIA for EIA and SEA is studies. Eco-indicator 99, IMPACT 2002+ and ReCiPe are the LCIA tools used in this study. Finally, a Taiwanese naphtha cracking plant is used as the example for an EIA and the Taiwanese solid waste policy as the case study for a SEA, in order to demonstrate the use of the proposed methodology.
\end{abstract}

Index Terms - Environmental impact assessment, strategic environmental assessment, life cycle impact assessment.

\section{INTRODUCTION}

An environmental impact assessment (EIA) is an environmental management plan for which scientific, objective and comprehensive surveys, forecasting, analysis and evaluations are conducted prior to project implementation, in order to determine the degree and scope of the potential impact of development activity or government policy on the environment, society, economy, culture and ecology of an area, and the public explanation and review of the plan. In Taiwan, development projects for which there is concern of an adverse impact on the environment must submit an environmental impact statement for the phase-I EIA and forward this environmental impact statement to a competent authority, for review. The developer must produce an environmental impact assessment report for the phase-II EIA, for those circumstances in which the result of the review of the environmental impact statement show concerns for a significant impact on the environment. The results of the review of environmental impact statements or environmental impact assessment reports are classified into three categories: Conditional approval, phase-II EIA, or rejection of the development plan [1].

In Taiwan, environmental impact statements and environmental impact assessment reports must evaluate the impact on the following environmental aspects:

Manuscript received November 15, 2014; revised January 13, 2015.

K. F. R. Liu, S. Y. Chiu and P. C. Yeh are with the Department of Safety, Health and Environmental Engineering, Ming Chi University of Technology, New Taipei City, Taiwan 24301, ROC (e-mail: kevinliu@mail.mcut.edu.tw).

J. Y. Kuo is with the Department of Science and Information Engineering, National Taipei University of Technology, Taipei, Taiwan 10608, ROC (e-mail: jykuo@ntut.edu.tw).
1) Physical and chemical environment

- Topography, geology and soil

- Water

- Climate and air quality

- Noise

- Vibration

- Odor

- Waste

- Excavation

- Coverage

- $\quad$ Energy

- Radiation

2) Ecology

- Terrestrial

- Aquatic

- $\quad$ Endangered species

- $\quad$ Ecosystems

3) Landscape \& Recreation

- $\quad$ Scenic beauty

- Recreation

4) Society \& Economy

- $\quad$ Land Use

- Social environment

- Transportation

- Economic welfare

- Social relationships

5) Culture

- $\quad$ Educational, scientific

- Historic, monumental

- Cultural

A strategic environmental assessment (SEA) is a systematic process for evaluating the environmental consequences of proposed policies, plans or programs of initiatives, in order to ensure they are fully included and appropriately addressed at the earliest appropriate stage of decision making and are given a similar level of importance to economic and social considerations [2].

In Taiwan, the SEA and EIA procedures are very similar. However, the impact considered in SEA reports is more extensive, as follows:

1) Assimilative capacity of the environment

$\begin{array}{ll}\text { - } & \text { Air } \\ \text { - } & \text { Water } \\ \text { - } & \text { Wail } \\ \text { - } & \text { Noise } \\ \text { - } & \text { Non-ionizing radiation }\end{array}$

2) Natural ecology and landscape

- Terrestrial ecosystems

- Aquatic ecosystems

- $\quad$ Ecology of the landscape \& habitat 
3) Human Health and Safety

- Toxic or harmful substances

- Risk of ionizing radiation

- $\quad$ Risk of chemical substances

4) Land resource

- Characteristics of land resources

- Mineral resource \& debris

- Land use

- Landscape

5) Water resources

- Distribution of water use

- Water crowding effect

- Water resources

6) Cultural assets

7) International environmental regulations as defined by the following:

- The Montreal Protocol

- The Framework Convention on Climate Change

- The Basel Convention

- The Washington Convention

- The Convention on Biological Diversity

- The Ramsar Convention on Wetlands

- The Stockholm Convention

- The Rotterdam Convention

8) Society \& Economy

- $\quad$ Population \& Industry

- Transportation

- $\quad$ Energy use

- $\quad$ Economic benefits

- $\quad$ Public facilities \& community development

- $\quad$ Public opinion \& social acceptance

EIAs and SEAs are procedural tools for environmental management that identify, predict, evaluate and mitigate the environmental impact of development proposals or policies. Many analytical tools improve the analysis of environmental impact in EIA or SEA reports, such as a life cycle assessment (LCA). A life cycle impact assessment (LCIA) is one of steps in a LCA that takes account of the causal relationships between environmental hazards and damage. Using a LCIA to produce EIA and SEA reports extends the focus of the reports from regulatory compliance for environmental impact, to determining the significance of the environmental impact [3].

\section{MethodS AND MATERIALS}

\section{A. Life Cycle Impact Assessment}

A life-cycle assessment assesses the environmental impact that is associated with all of the stages of a product's life, from raw material extraction through material processing, manufacture, distribution, use, repair and maintenance, and disposal or recycling. According to the ISO 14040 [4] and 14044 [5] standards, a Life Cycle Assessment has four distinct phases. The first phase is the 'Goal and scope', which requires an explicit statement of the goal and scope of the study. It establishes the context of the study and explains how and to whom the results are to be communicated. The second phase is a 'Life cycle inventory (LCI)', which involves the creation of an inventory of the flows from and to nature for a product system. Inventory flows include inputs of water, energy and raw materials, and releases to air, land and water. The third phase is a 'Life cycle impact assessment (LCIA)', which evaluates the significance of any potential environmental impact, based on the results for the LCI flow. A classical LCIA involves selection of the following mandatory elements: impact categories, category indicators and characterization models. In the classification stage, the inventory parameters are categorized and assigned to specific impact categories. In the impact measurement stage, the categorized LCI flows are characterized into common equivalence units, using one of many possible LCIA methodologies. These are then summed to provide a total overall impact. The last phase is 'Interpretation', which is a systematic technique that identifies, quantifies, checks and evaluates information from the results of the life cycle inventory and/or the life cycle impact assessment. The results of the inventory analysis and the impact assessment are summarized during the interpretation phase.

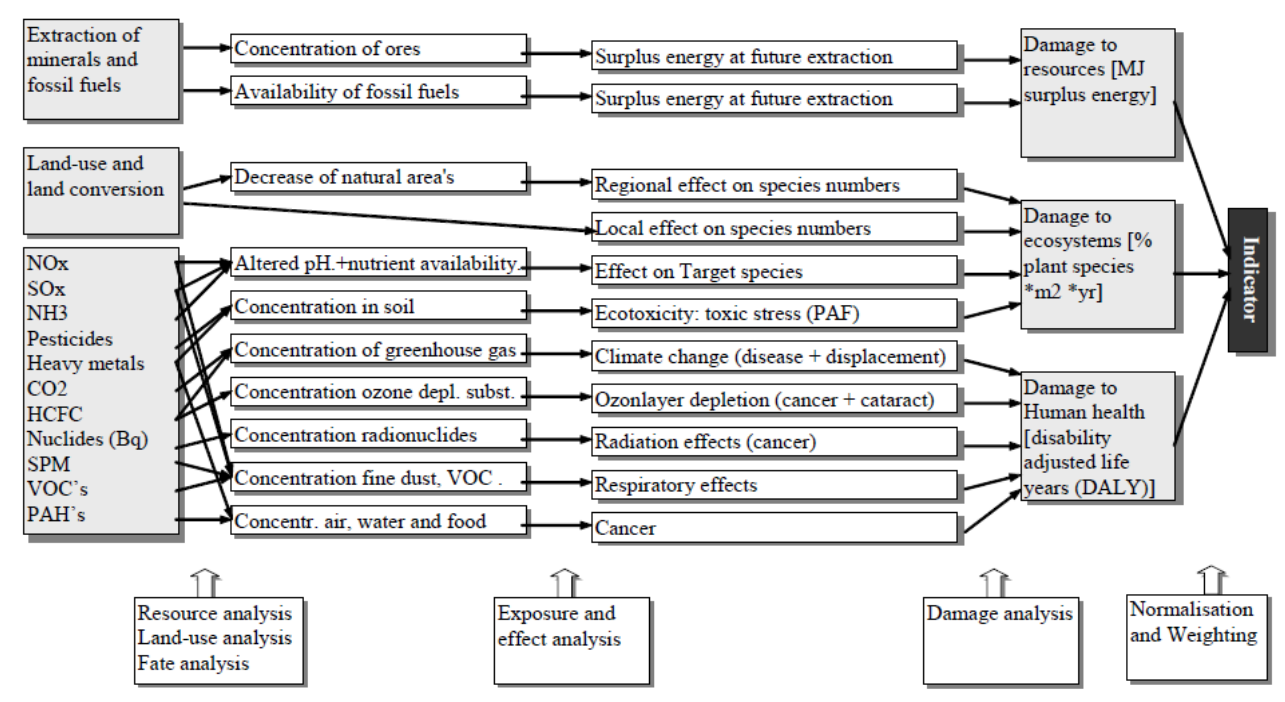

Fig. 1. The framework of Eco-indicator 99 [9].

Three LCIA methods are used for this study: Eco-indicator 99 [6], IMPACT 2002+ [7] and ReCiPe [8]. Eco-indicator 99 (Fig. 1) is one of the most widely used impact assessment methods for a LCA. It is the successor of Eco-indicator 95, the first endpoint impact assessment method, which allows the environmental load of a product to be expressed in a 
single score. The Life Cycle Impact Assessment methodology, IMPACT 2002+ (Fig. 2), suggests a feasible implementation of a combined midpoint/damage approach. These combinations link all types of Life Cycle Inventory (LCI) results, the elementary flows and other interventions, throughout the 14 midpoint categories, which are summed into four damage categories. ReCiPe (Fig. 3) is a fusion of these two methodologies, taking the midpoint indicators from $\mathrm{CML}$ and the endpoint indicators from Ecoindicator.

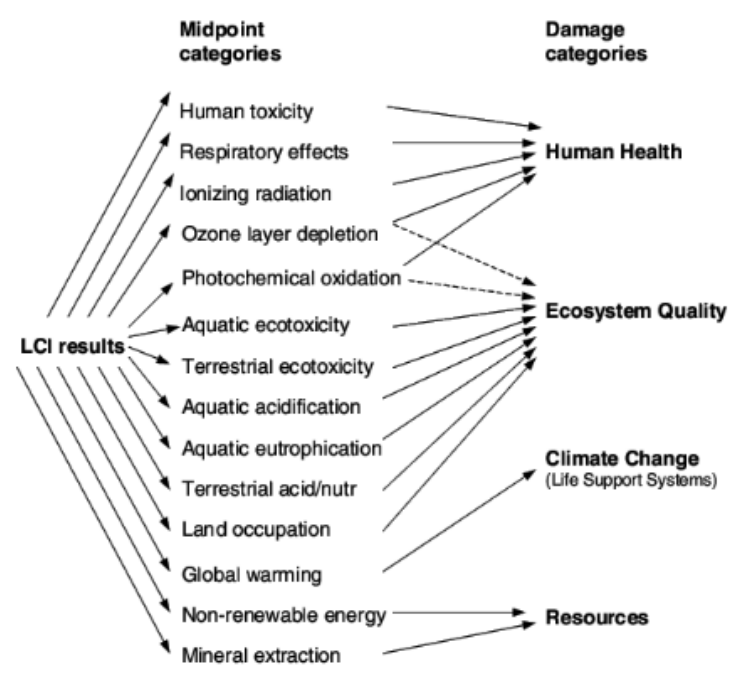

Fig. 2. The framework of IMPACT 2002+ [7].

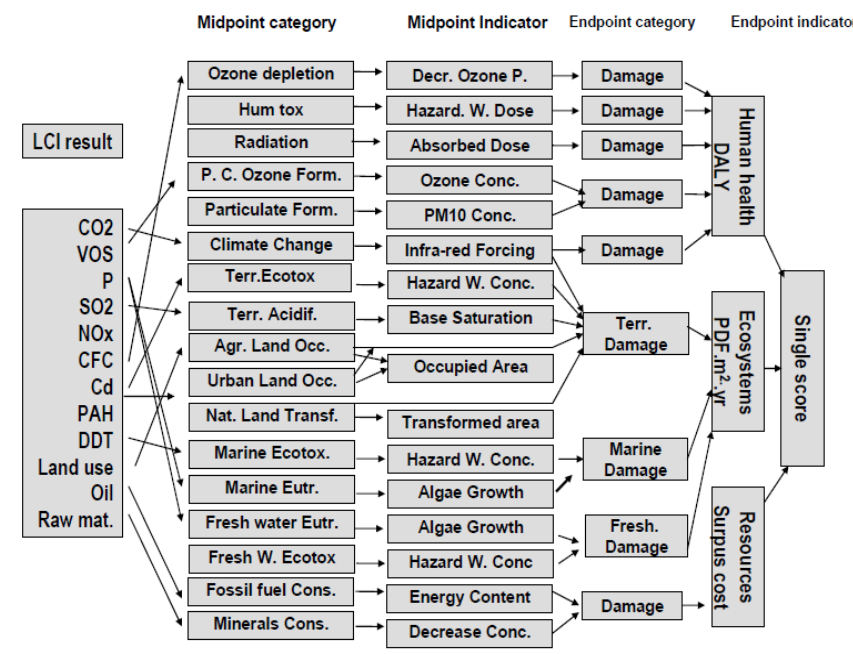

Fig. 3. The framework of ReCiPe [9].

\section{B. Case Study (1)}

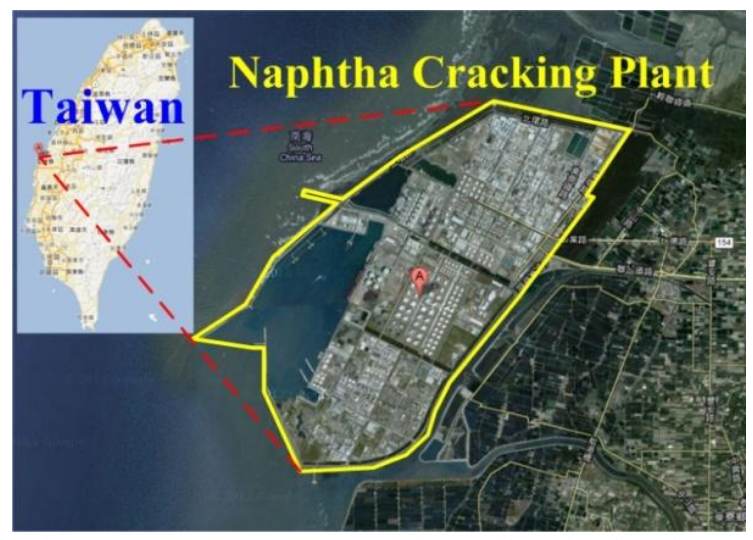

Fig. 4. The case study (1): A naphtha cracking plant in Taiwan.
TABLE I: THE INVENTORY DATA FOR CASE STUDY (1)

\begin{tabular}{|c|c|c|c|c|}
\hline Category & Inventory Item & $\mathrm{BE}$ & $\mathrm{AE}$ & Unit \\
\hline Resource & Water & $1.261 \mathrm{E}+02$ & $1.736 \mathrm{E}+02$ & Mton \\
\hline \multirow{37}{*}{ Air } & Total Suspended Particulate(TSP) & $3.340 \mathrm{E}+00$ & $4.320 \mathrm{E}+00$ & kton \\
\hline & particulate matter (PM10) & $1.690 \mathrm{E}+00$ & $2.160 \mathrm{E}+00$ & kton \\
\hline & Sulfur oxide(SOx) & $1.600 \mathrm{E}+01$ & $1.979 \mathrm{E}+01$ & kton \\
\hline & Nitrogen oxide(NOx) & $1.962 \mathrm{E}+01$ & $2.388 \mathrm{E}+01$ & kton \\
\hline & $\begin{array}{l}\text { Volatile organic } \\
\text { compounds(VOCs) }\end{array}$ & $4.300 \mathrm{E}+00$ & $5.390 \mathrm{E}+00$ & kton \\
\hline & Carbon dioxide & $6.757 \mathrm{E}+01$ & $7.815 \mathrm{E}+01$ & Mton \\
\hline & Butadiene & $1.355 \mathrm{E}+03$ & $1.723 \mathrm{E}+03$ & $\mathrm{~kg}$ \\
\hline & Benzene & $3.969 \mathrm{E}+02$ & $5.046 \mathrm{E}+02$ & $\mathrm{~kg}$ \\
\hline & Ethylene oxide & $2.634 \mathrm{E}+02$ & $3.349 \mathrm{E}+02$ & $\mathrm{~kg}$ \\
\hline & Formaldehyde & $4.006 \mathrm{E}+02$ & $5.093 \mathrm{E}+02$ & $\mathrm{~kg}$ \\
\hline & Ethene, chloro- & $2.799 \mathrm{E}+02$ & $3.558 \mathrm{E}+02$ & $\mathrm{~kg}$ \\
\hline & Ethene, trichloro- & $9.820 \mathrm{E}+00$ & $1.249 \mathrm{E}+01$ & $\mathrm{~kg}$ \\
\hline & Acetaldehyde & $6.256 \mathrm{E}+01$ & $7.953 \mathrm{E}+01$ & $\mathrm{~kg}$ \\
\hline & Acrylonitrile & $6.622 \mathrm{E}+02$ & $8.418 \mathrm{E}+02$ & $\mathrm{~kg}$ \\
\hline & Methane, tetrachloro-, CFC-10 & $1.151 \mathrm{E}+01$ & $1.463 \mathrm{E}+01$ & $\mathrm{~kg}$ \\
\hline & Chloroform & $1.340 \mathrm{E}+00$ & $1.700 \mathrm{E}+00$ & $\mathrm{~kg}$ \\
\hline & Ethyl acrylate & $3.750 \mathrm{E}+01$ & $4.767 \mathrm{E}+01$ & $\mathrm{~kg}$ \\
\hline & Benzene, ethyl- & $4.774 \mathrm{E}+01$ & $6.069 \mathrm{E}+01$ & $\mathrm{~kg}$ \\
\hline & Ethane, 1,2-dichloro- & $8.890 \mathrm{E}+01$ & $1.130 \mathrm{E}+02$ & $\mathrm{~kg}$ \\
\hline & Naphthalene & $1.921 \mathrm{E}+01$ & $2.442 \mathrm{E}+01$ & $\mathrm{~kg}$ \\
\hline & Benzene, 1,4-dichloro- & $1.099 \mathrm{E}+02$ & $1.398 \mathrm{E}+02$ & $\mathrm{~kg}$ \\
\hline & Propylene oxide & $4.353 \mathrm{E}+02$ & $5.535 \mathrm{E}+02$ & $\mathrm{~kg}$ \\
\hline & Styrene & $1.247 \mathrm{E}+03$ & $1.586 \mathrm{E}+03$ & $\mathrm{~kg}$ \\
\hline & Vinyl acetate & $8.377 \mathrm{E}+01$ & $1.065 \mathrm{E}+02$ & $\mathrm{~kg}$ \\
\hline & Acrolein & $5.872 \mathrm{E}+01$ & $7.465 \mathrm{E}+01$ & $\mathrm{~kg}$ \\
\hline & Methane, monochloro-, R-40 & $1.776 \mathrm{E}+02$ & $2.258 \mathrm{E}+02$ & $\mathrm{~kg}$ \\
\hline & Methanol & $7.024 \mathrm{E}+02$ & $8.930 \mathrm{E}+02$ & $\mathrm{~kg}$ \\
\hline & Carbon disulfide & $1.140 \mathrm{E}+01$ & $1.449 \mathrm{E}+01$ & $\mathrm{~kg}$ \\
\hline & Hexane & $7.280 \mathrm{E}+02$ & $9.255 \mathrm{E}+02$ & $\mathrm{~kg}$ \\
\hline & t-Butyl methyl ether & $5.268 \mathrm{E}+01$ & $6.697 \mathrm{E}+01$ & $\mathrm{~kg}$ \\
\hline & Xylene & $9.603 \mathrm{E}+02$ & $1.221 \mathrm{E}+03$ & $\mathrm{~kg}$ \\
\hline & Methyl methacrylate & $3.073 \mathrm{E}+02$ & $3.907 \mathrm{E}+02$ & $\mathrm{~kg}$ \\
\hline & Ethane, chloro- & $4.792 \mathrm{E}+01$ & $6.093 \mathrm{E}+01$ & $\mathrm{~kg}$ \\
\hline & Methyl ethyl ketone & $1.079 \mathrm{E}+02$ & $1.372 \mathrm{E}+02$ & $\mathrm{~kg}$ \\
\hline & Toluene & $1.310 \mathrm{E}+02$ & $1.665 \mathrm{E}+02$ & $\mathrm{~kg}$ \\
\hline & Benzene, chloro- & $1.010 \mathrm{E}+02$ & $1.284 \mathrm{E}+02$ & $\mathrm{~kg}$ \\
\hline & Dimethyl formamide & $1.530 \mathrm{E}+00$ & $1.950 \mathrm{E}+00$ & $\mathrm{~kg}$ \\
\hline \multirow[t]{28}{*}{ Water } & Wastewater & $6.862 \mathrm{E}+01$ & $1.111 \mathrm{E}+02$ & Mton \\
\hline & suspended solids & $8.400 \mathrm{E}-01$ & $1.360 \mathrm{E}+00$ & kton \\
\hline & Chloride & $1.240 \mathrm{E}+00$ & $2.000 \mathrm{E}+00$ & Mton \\
\hline & Fluoride & $8.715 \mathrm{E}+01$ & $1.412 \mathrm{E}+02$ & ton \\
\hline & Sulfate & $1.900 \mathrm{E}-01$ & $3.100 \mathrm{E}-01$ & Mton \\
\hline & Sulfide & $1.850 \mathrm{E}+00$ & $3.000 \mathrm{E}+00$ & ton \\
\hline & Chlorine & $6.180 \mathrm{E}+00$ & $1.000 \mathrm{E}+01$ & ton \\
\hline & Biochemical oxygen demand & $3.637 \mathrm{E}+02$ & $5.891 \mathrm{E}+02$ & ton \\
\hline & Chemical oxygen demand & $2.240 \mathrm{E}+00$ & $3.630 \mathrm{E}+00$ & kton \\
\hline & Ammonia, as $\mathrm{N}$ & $1.022 \mathrm{E}+02$ & $1.656 \mathrm{E}+02$ & ton \\
\hline & Nitrate & $4.783 \mathrm{E}+02$ & $7.747 \mathrm{E}+02$ & ton \\
\hline & Nitrite & $1.320 \mathrm{E}+00$ & $2.130 \mathrm{E}+00$ & kton \\
\hline & Phosphate & $6.860 \mathrm{E}+00$ & $1.111 \mathrm{E}+01$ & ton \\
\hline & Phosphorus, TOTAL & $1.510 \mathrm{E}+01$ & $2.445 \mathrm{E}+01$ & ton \\
\hline & Oils, unspecified & $4.117 \mathrm{E}+01$ & $6.669 \mathrm{E}+01$ & ton \\
\hline & Cyanide & $6.200 \mathrm{E}-01$ & $1.000 \mathrm{E}+00$ & ton \\
\hline & Phenols, unspecified & $1.240 \mathrm{E}+00$ & $2.000 \mathrm{E}+00$ & ton \\
\hline & Arsenic & $1.098 \mathrm{E}+01$ & $1.778 \mathrm{E}+01$ & ton \\
\hline & cadmium & $9.600 \mathrm{E}-01$ & $1.560 \mathrm{E}+00$ & ton \\
\hline & Chromium & $8.230 \mathrm{E}+00$ & $1.334 \mathrm{E}+01$ & ton \\
\hline & Copper & $3.430 \mathrm{E}+00$ & $5.560 \mathrm{E}+00$ & ton \\
\hline & Mercury & $7.000 \mathrm{E}-02$ & $1.100 \mathrm{E}-01$ & ton \\
\hline & Nickel & $6.180 \mathrm{E}+00$ & $1.000 \mathrm{E}+01$ & ton \\
\hline & Lead & $1.304 \mathrm{E}+01$ & $2.112 \mathrm{E}+01$ & ton \\
\hline & Selenium & $1.029 \mathrm{E}+01$ & $1.667 \mathrm{E}+01$ & ton \\
\hline & Zinc & $1.098 \mathrm{E}+01$ & $1.778 \mathrm{E}+01$ & ton \\
\hline & Iron & $6.180 \mathrm{E}+00$ & $1.000 \mathrm{E}+01$ & ton \\
\hline & Manganese & $3.430 \mathrm{E}+00$ & $5.560 \mathrm{E}+00$ & ton \\
\hline \multirow[t]{5}{*}{ Waste } & Incineration waste in incineration & $1.532 \mathrm{E}+05$ & $1.541 \mathrm{E}+05$ & ton \\
\hline & Landfill waste in incineration & $5.253 \mathrm{E}+04$ & $9.009 \mathrm{E}+04$ & ton \\
\hline & waste to recycling & $1.893 \mathrm{E}+06$ & $3.910 \mathrm{E}+06$ & ton \\
\hline & Sludge & $3.095 \mathrm{E}+05$ & $0.000 \mathrm{E}+00$ & ton \\
\hline & Coal ash & $1.175 \mathrm{E}+06$ & $0.000 \mathrm{E}+00$ & ton \\
\hline
\end{tabular}

The case study is a naphtha cracking plant that is located in Yunlin County, in Taiwan, as shown in Fig. 4. It is in an 
offshore industrial zone, with a total area of 2,603 hectares. Currently, as alternative BE (before expansion), 61 factories have an annual output of 6,221 tons. In response to market demand, the company proposes an expansion plan (alternative AE, after expansion) that will increase the number of factories to 77 and increase production to 8,174 tons per year, which is an increase of $31.4 \%$. However, the expansion plan will also increase its emissions of TSP from 3,340 to 4,323 tons per year, $\mathrm{SO}_{2}$ from 16,000 to 19,788 tons per year, $\mathrm{NO}_{2}$ from 19,622 to 23,881 tons per year, VOC from 4,302 to 5,389 tons per year and waste-water from 188,000 to 304,500 tons per day. Its inventory flows, including inputs of water, energy and raw materials and releases to air, land, and water, are detailed in Table I.

\section{Case Study (2)}

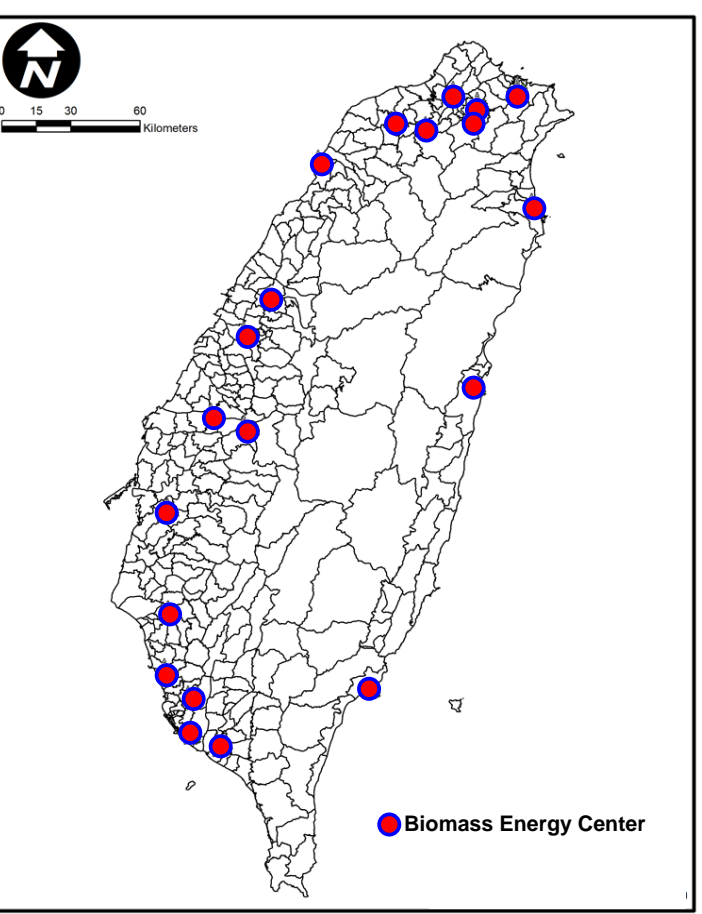

Fig. 5. The case study (2): 20 biomass energy centers in Taiwan.

TABLE II: THE INVENTORY DATA FOR CASE STUDY (2)

\begin{tabular}{|c|c|c|c|c|c|}
\hline Category & Inventory Item & $\overline{Z Z}$ & $\overline{\mathrm{A}}$ & $\overline{\mathrm{B}}$ & Unit \\
\hline \multirow[t]{2}{*}{ Products } & Electricity & $1.877 \mathrm{E}+09$ & $1.708 \mathrm{E}+09$ & $3.017 \mathrm{E}+09$ & $\mathrm{kWh}$ \\
\hline & Bio-coal & & $1.042 \mathrm{E}+06$ & & ton \\
\hline Resources & Water & $2.256 \mathrm{E}+06$ & $1.615 \mathrm{E}+06$ & $2.652 \mathrm{E}+06$ & $\mathrm{~m}^{3}$ \\
\hline Fuels & Diesel, at refinery/l/US & $2.097 \mathrm{E}+06$ & $3.606 \mathrm{E}+08$ & $3.497 \mathrm{E}+08$ & $\mathrm{~L}$ \\
\hline Electricity & Electricity & $4.769 \mathrm{E}+05$ & $2.775 \mathrm{E}+08$ & $1.252 \mathrm{E}+09$ & $\mathrm{kWh}$ \\
\hline \multirow[t]{10}{*}{ Air } & Sulfur oxides & $3.430 \mathrm{E}+06$ & $6.011 \mathrm{E}+05$ & $4.591 \mathrm{E}+05$ & $\mathrm{~kg}$ \\
\hline & Nitrogen oxides & $6.429 \mathrm{E}+06$ & $1.213 \mathrm{E}+06$ & $5.916 \mathrm{E}+06$ & $\mathrm{~kg}$ \\
\hline & TSP & $3.834 \mathrm{E}+06$ & $1.068 \mathrm{E}+05$ & $1.298 \mathrm{E}+05$ & $\mathrm{~kg}$ \\
\hline & Arsenic & $1.130 \mathrm{E}+02$ & $1.300 \mathrm{E}+01$ & 4.600E+00 & $\mathrm{kg}$ \\
\hline & Cadmium & $2.930 \mathrm{E}+01$ & $2.900 \mathrm{E}+01$ & $2.950 \mathrm{E}+01$ & $\mathrm{~kg}$ \\
\hline & Chromium & $2.420 \mathrm{E}+02$ & $1.360 \mathrm{E}+02$ & $3.620 \mathrm{E}+01$ & $\mathrm{~kg}$ \\
\hline & Lead & $5.410 \mathrm{E}+02$ & $6.100 \mathrm{E}+01$ & $9.220 \mathrm{E}+02$ & $\mathrm{~kg}$ \\
\hline & $\begin{array}{l}\text { Dioxin, } \\
1,2,3,7,8,9 \text {-hexachlorodibenzo- }\end{array}$ & $1.000 \mathrm{E}-03$ & $6.430 \mathrm{E}-05$ & $5.120 \mathrm{E}-04$ & $\mathrm{~kg}$ \\
\hline & Carbon monoxide & $2.150 \mathrm{E}+04$ & $1.910 \mathrm{E}+04$ & $2.910 \mathrm{E}+04$ & $\mathrm{~kg}$ \\
\hline & Carbon dioxide & $3.660 \mathrm{E}+05$ & $3.030 \mathrm{E}+05$ & $3.410 \mathrm{E}+05$ & ton \\
\hline \multirow[t]{9}{*}{ Water } & Suspended solids, unspecified & & $2.910 \mathrm{E}+04$ & & $\mathrm{~kg}$ \\
\hline & $\begin{array}{l}\text { BOD5, Biological Oxygen } \\
\text { Demand }\end{array}$ & & $2.910 \mathrm{E}+04$ & & $\mathrm{~kg}$ \\
\hline & COD, Chemical Oxygen & & $9.690 \mathrm{E}+04$ & & $\mathrm{~kg}$ \\
\hline & Demand & & & & \\
\hline & Chloride & & $4.840 \mathrm{E}+02$ & & $\mathrm{~kg}$ \\
\hline & Arsenic & & $4.840 \mathrm{E}+02$ & & $\mathrm{~kg}$ \\
\hline & Cadmium & & $2.910 \mathrm{E}+01$ & & $\mathrm{~kg}$ \\
\hline & Chromium & & $1.940 \mathrm{E}+03$ & & $\mathrm{~kg}$ \\
\hline & Lead & & $9.690 \mathrm{E}+02$ & & $\mathrm{~kg}$ \\
\hline \multirow[t]{2}{*}{ Waste } & Waste, inorganic & & $5.893 \mathrm{E}+04$ & $8.880 \mathrm{E}+05$ & ton \\
\hline & Fly ash & $2.711 \mathrm{E}+04$ & & & ton \\
\hline
\end{tabular}

In Taiwan, about 3.5 million tons of biomass garbage is generated each year, with a potential energy of more than 100 tons of natural coal. At present, a total of 24 incineration plants are in operation and their power generation efficiency is only $20 \%$, which is much lower than $35 \%$ for coal-fired plants. The current incineration of solid waste is the reference point, zero alternative (Z). Two policy alternatives, (A) and (B), are proposed, to improve the efficiency of power generation and thereby allow the integration of energy and resource, sustainable recycling, energy saving and carbon reduction. Alternative (A) transforms the current incineration plants into 20 biomass energy centers (Fig. 5) by using new waste and biomass energy utilization technologies. Alternative (B) transforms the current incineration plants by integrating refuse derived fuel plants (RDF) and RDF burning power plants. Their inventory flows, including inputs of water, energy and raw materials and releases to air, land and water, are detailed in Table II.

\section{RESUlts}

\section{A. LCIA for EIA: Case Study (1)}

TABLE III: THE USE OF LCIA TOOLS FOR EIA EVALUATION ITEMS

\begin{tabular}{|c|c|c|c|}
\hline EIA Evaluation Item & Eco-indicator99 & IMPACT 2002+ & $\mathrm{ReCiPe}$ \\
\hline \multirow{3}{*}{$\begin{array}{l}\text { (A) Physical and chemical } \\
\text { - Topography, geology \& } \\
\text { soil }\end{array}$} & & & \\
\hline & Acidification/ & $\begin{array}{l}\text { Terrestrial } \\
\text { acid/nutri }\end{array}$ & Terrestrial acidification \\
\hline & Minerals & Mineral extraction & Metal depletion \\
\hline \multirow[t]{2}{*}{ •Water } & Acidification/ & Aquatic & \\
\hline & Eutrophication & acidification & \\
\hline \multirow[t]{4}{*}{-Climate and air quality } & Ozone layer & $\begin{array}{l}\text { Ozone layer } \\
\text { depletion }\end{array}$ & Ozone depletion \\
\hline & Respiratory organics & $\begin{array}{l}\text { Respiratory } \\
\text { organics }\end{array}$ & $\begin{array}{l}\text { Photochemical oxidant } \\
\text { formation }\end{array}$ \\
\hline & Respiratory inorganics & $\begin{array}{l}\text { Respiratory } \\
\text { inorganics }\end{array}$ & Particulate matter formation \\
\hline & Climate change & Global warming & Climate change Human Health \\
\hline \multicolumn{4}{|c|}{ 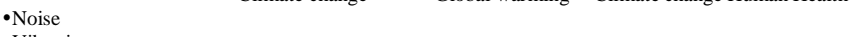 } \\
\hline \multicolumn{4}{|l|}{-Vibration } \\
\hline \multicolumn{4}{|l|}{ •Odor } \\
\hline \multicolumn{4}{|l|}{-Waste } \\
\hline \multicolumn{4}{|l|}{-Excavation } \\
\hline \multicolumn{4}{|l|}{ - Coverage } \\
\hline •Energy & Fossil fuels & $\begin{array}{l}\text { Non-renewable } \\
\text { energy }\end{array}$ & Fossil depletion \\
\hline - Radiation & Radiation & Ionizing radiation & Ionising radiation \\
\hline (B) Ecology & & & Climate change Ecosystems \\
\hline •Terrestrial & Ecotoxicity & $\begin{array}{l}\text { Terrestrial } \\
\text { ecotoxicity }\end{array}$ & Terrestrial ecotoxicity \\
\hline •Aquatic & Ecotoxicity & $\begin{array}{l}\text { Aquatic } \\
\text { ecotoxicity }\end{array}$ & Freshwater ecotoxicity \\
\hline \multicolumn{4}{|l|}{ •Endangered } \\
\hline •Ecosystem & $\begin{array}{l}\text { Acidification/ } \\
\text { Eutrophication }\end{array}$ & $\begin{array}{l}\text { Aquatic } \\
\text { eutrophication }\end{array}$ & Freshwater eutrophication \\
\hline \multicolumn{4}{|l|}{ (C) Landscape \& } \\
\hline \multicolumn{4}{|l|}{ Recreation } \\
\hline \multicolumn{4}{|l|}{-Scenic beauty } \\
\hline \multicolumn{4}{|l|}{$\begin{array}{l}\text { - Recreation } \\
\text { (D) Society \& Economy }\end{array}$} \\
\hline $\begin{array}{l}\text { (D) Society \& Economy } \\
\text {-Land Use }\end{array}$ & Land use & Land occupation & $\begin{array}{l}\text { Urban land occupation } \\
\text { Agricultural land occupation } \\
\text { Natural land transformation }\end{array}$ \\
\hline \multicolumn{4}{|l|}{-Social environment } \\
\hline \multicolumn{4}{|l|}{ - Transportation } \\
\hline \multicolumn{4}{|l|}{ •Economic level } \\
\hline \multicolumn{4}{|l|}{$\begin{array}{l}\text {-Social relationships } \\
\text { (E) Culture }\end{array}$} \\
\hline \multicolumn{4}{|l|}{ (E) Culture } \\
\hline \multicolumn{4}{|l|}{-Educational, scientific } \\
\hline \multicolumn{4}{|l|}{-Historic, monumental } \\
\hline -Cultural & & & \\
\hline
\end{tabular}

In Taiwan, evaluation items for an EIA are: (1) physical and chemical, including topography-geology-soil, water, climate and air quality, noise, vibration, odor, waste, excavation, coverage, energy and radiation; (2) ecology, including terrestrial, aquatic, endangered and ecosystem; (3) landscape \& recreation, including scenic beauty and recreation; (4) society \& economy, including land use, social environment, transportation, economic level and social 
relationships; (5) culture, including educational, scientific, historic, monumental and cultural. Table III summarizes the use of the three LCIA methods, Eco-indicator 99, IMPACT $2002+$ and ReCiPe, to assess evaluation items in the EIA. Some items are not evaluated by the three LCIA methods such as noise, vibration, odor, waste, excavation, coverage, endangered, scenic beauty, recreation, social environment, transportation, economic level, social relationships, educational, scientific, historic, monumental and cultural.

TABLE IV: THE RESULTS FOR THE USE OF LCIA TOOLS FOR CASE STUDY (1)

\begin{tabular}{|c|c|c|c|c|}
\hline \multicolumn{5}{|c|}{ Eco-indicator 99} \\
\hline Impact Category & Unit & $\mathrm{BE}$ & $\mathrm{AE}$ & Increase \\
\hline Carcinogens & DALY & $1.030 \mathrm{E}-01$ & $1.309 \mathrm{E}-01$ & $+27.1 \%$ \\
\hline Respiratory organics & DALY & $2.785 \mathrm{E}+00$ & $3.491 \mathrm{E}+00$ & $+25.4 \%$ \\
\hline Respiratory inorganics & DALY & $3.255 \mathrm{E}+03$ & $4.018 \mathrm{E}+03$ & $+23.4 \%$ \\
\hline Climate change & DALY & $1.419 \mathrm{E}+04$ & $1.641 \mathrm{E}+04$ & $+15.6 \%$ \\
\hline Radiation & DALY & & & \\
\hline Ozone layer & DALY & $1.825 \mathrm{E}-02$ & $2.320 \mathrm{E}-02$ & $+27.1 \%$ \\
\hline Ecotoxicity & $\mathrm{PDF}^{*} \mathrm{~m}^{2} * \mathrm{yr}$ & $6.756 \mathrm{E}+06$ & $1.094 \mathrm{E}+07$ & $+61.9 \%$ \\
\hline Acidification/ Eutrophication & $\mathrm{PDF}^{*} \mathrm{~m}^{2} * \mathrm{yr}$ & $1.287 \mathrm{E}+08$ & $1.570 \mathrm{E}+08$ & $+22.0 \%$ \\
\hline Land use & $\mathrm{PDF}^{*} \mathrm{~m}^{2} * \mathrm{yr}$ & & & \\
\hline Minerals & MJ surplus & & & \\
\hline Fossil fuels & MJ surplus & & & \\
\hline Human Health & DÁYY & $1.745 \mathrm{E}+04$ & $2.043 \mathrm{E}+04$ & $+17.1 \%$ \\
\hline Ecosystem Quality & $\mathrm{PDF}^{*} \mathrm{~m}^{2} \mathrm{yr}$ & $1.355 \mathrm{E}+08$ & $1.679 \mathrm{E}+08$ & $+23.9 \%$ \\
\hline Resources & MJ surplus & & & \\
\hline \multicolumn{5}{|c|}{ IMPACT $2002+$} \\
\hline Impact Category & Unit & $\mathrm{BE}$ & $\mathrm{AE}$ & Increase \\
\hline Carcinogens & DALY & $3.561 \mathrm{E}+03$ & $4.528 \mathrm{E}+03$ & $+27.2 \%$ \\
\hline Non-carcinogens & DALY & $1.132 \mathrm{E}+07$ & $1.834 \mathrm{E}+07$ & $+62.0 \%$ \\
\hline Respiratory inorganics & DALY & $5.176 \mathrm{E}+06$ & $6.419 \mathrm{E}+06$ & $+24.0 \%$ \\
\hline Ionizing radiation & DALY & & & \\
\hline Ozone layer depletion & DALY & $1.195 \mathrm{E}+01$ & $1.520 \mathrm{E}+01$ & $+27.2 \%$ \\
\hline Respiratory organics & DALY & $1.307 \mathrm{E}+06$ & $1.639 \mathrm{E}+06$ & $+25.4 \%$ \\
\hline Aquatic ecotoxicity & $\mathrm{PDF}^{*} \mathrm{~m}^{2} * \mathrm{yr}$ & $5.869 \mathrm{E}+10$ & $9.501 \mathrm{E}+10$ & $+61.9 \%$ \\
\hline Terrestrial ecotoxicity & $\mathrm{PDF}^{*} \mathrm{~m}^{2} * \mathrm{yr}$ & $6.481 \mathrm{E}+03$ & $8.239 \mathrm{E}+03$ & $+27.1 \%$ \\
\hline Terrestrial acid/nutri & $\mathrm{PDF}^{*} \mathrm{~m}^{2} * \mathrm{yr}$ & $1.237 \mathrm{E}+08$ & $1.508 \mathrm{E}+08$ & $+21.9 \%$ \\
\hline Land occupation & $\mathrm{PDF}^{*} \mathrm{~m}^{2} * \mathrm{yr}$ & & & \\
\hline Aquatic acidification & $\mathrm{PDF}^{*} \mathrm{~m}^{2} * \mathrm{yr}$ & $2.997 \mathrm{E}+07$ & $3.688 \mathrm{E}+07$ & $+23.1 \%$ \\
\hline Aquatic eutrophication & $\mathrm{PDF}^{*} \mathrm{~m}^{2} * \mathrm{yr}$ & $1.023 \mathrm{E}+05$ & $1.658 \mathrm{E}+05$ & $+62.1 \%$ \\
\hline Global warming & $\mathrm{kg} \mathrm{CO}_{2}$ eq & $6.757 \mathrm{E}+10$ & $7.815 \mathrm{E}+10$ & $+15.7 \%$ \\
\hline Non-renewable energy & MJ primary & & & \\
\hline Mineral extraction & MJ primary & & & \\
\hline Human Health & DALY & $1.782 \mathrm{E}+07$ & $2.642 \mathrm{E}+07$ & $+48.3 \%$ \\
\hline Ecosystem Quality & $\mathrm{PDF}^{*} \mathrm{~m}^{2} \mathrm{yr}$ & $5.884 \mathrm{E}+10$ & $9.520 \mathrm{E}+10$ & $+61.8 \%$ \\
\hline Resources & MJ surplus & & & \\
\hline \multicolumn{5}{|c|}{$\mathrm{ReCiPe}$} \\
\hline Impact Category & Unit & $\mathrm{BE}$ & $\mathrm{AE}$ & Increase \\
\hline Climate change Human Health & DALY & $2.372 \mathrm{E}+05$ & $2.743 \mathrm{E}+05$ & $+15.6 \%$ \\
\hline Ozone depletion & DALY & $2.774 \mathrm{E}-02$ & $3.526 \mathrm{E}-02$ & $+27.1 \%$ \\
\hline Human toxicity & DALY & $3.020 \mathrm{E}+04$ & $4.892 \mathrm{E}+04$ & $+62.0 \%$ \\
\hline $\begin{array}{l}\text { Photochemical oxidant } \\
\text { formation }\end{array}$ & DALY & $8.159 \mathrm{E}-01$ & $9.940 \mathrm{E}-01$ & $+21.8 \%$ \\
\hline Particulate matter formation & DALY & $2.394 \mathrm{E}+03$ & $2.957 \mathrm{E}+03$ & $+23.5 \%$ \\
\hline Ionising radiation & DALY & & & \\
\hline Climate change Ecosystems & species.yr & $1.264 \mathrm{E}+03$ & $1.461 \mathrm{E}+03$ & $+15.6 \%$ \\
\hline Terrestrial acidification & species.yr & $4.254 \mathrm{E}-01$ & $5.222 \mathrm{E}-01$ & $+22.8 \%$ \\
\hline Freshwater eutrophication & species.yr & $7.699 \mathrm{E}-04$ & $1.247 \mathrm{E}-03$ & $+62.0 \%$ \\
\hline Terrestrial ecotoxicity & species.yr & $1.574 \mathrm{E}-03$ & $2.485 \mathrm{E}-03$ & $+57.9 \%$ \\
\hline Freshwater ecotoxicity & species.yr & $2.670 \mathrm{E}-04$ & $4.324 \mathrm{E}-04$ & $+61.9 \%$ \\
\hline Marine ecotoxicity & species.yr & $9.640 \mathrm{E}-03$ & $1.562 \mathrm{E}-02$ & $+62.0 \%$ \\
\hline Agricultural land occupation & species.yr & & & \\
\hline Urban land occupation & species.yr & & & \\
\hline Natural land transformation & species.yr & & & \\
\hline Metal depletion & $\$$ & & & \\
\hline Fossil depletion & $\$$ & & & \\
\hline Human Health & DALY & $2.698 \mathrm{E}+05$ & $3.262 \mathrm{E}+05$ & $+20.9 \%$ \\
\hline Ecosystem Quality & species.yr & $1.264 \mathrm{E}+03$ & $1.462 \mathrm{E}+03$ & $+15.7 \%$ \\
\hline Resources & $\$$ & & & \\
\hline
\end{tabular}

The results for the use of Eco-indicator 99, IMPACT 2002+ and ReCiPe to assess case study (1) are shown in Table IV. In Table IV, The results evaluated by Eco-indicator 99 show the most negative impact on human health is 'climate change', and the most negative impact on ecosystem quality is 'acidification/ eutrophication'. The results evaluated by IMPACT 2002+ show the most negative impact on human health is 'non-carcinogens', and the most negative impact on ecosystem quality is 'Aquatic ecotoxicity'. The results evaluated by ReCiPe show the most negative impact on human health is 'climate change human health', and the most negative impact on ecosystem quality is 'Climate change ecosystems'. Compared to each other, the magnitude of impact evaluated by the three LCIA methods differs up to three orders for human health and two orders for ecosystem quality. For example, as for AE the impact of human health is 2.043E+04 DALY by Eco-indicator 99, but it raises up to 2.642E+07 DALY by IMPACT 2002+. Meanwhile, as for $\mathrm{AE}$ the impact of ecosystem quality is $1.679 \mathrm{E}+08 \mathrm{PDF}^{*} \mathrm{~m}^{2} \mathrm{yr}$ by Eco-indicator 99, but it turns into $9.520 \mathrm{E}+10 \mathrm{PDF}^{*} \mathrm{~m}^{2} \mathrm{yr}$ by IMPACT $2002+$. The difference among LCIA methods primarily comes from their inconsistent characterization models and it makes users confused because they use different models, geographic conditions, time horizons and receptors etc.

\section{B. LCIA for EIA: Case Study (2)}

In Taiwan, evaluation items for an SEA are: (1) assimilative capacity of the environment, including air, water, soil, waste, noise and non-ionizing radiation; (2) natural ecology and landscape, including terrestrial ecosystems, aquatic ecosystems and ecological landscape \& habitat; (3) human health and safety, including toxic or harmful substances, risk of ionizing radiation and risk of chemical substances; (4) land resource, including characteristics of land resources, mineral resource \& debris, land use and landscape; (5) water resource, including distribution of water use, water crowding effect and water resource; (6) cultural assets; (7) international environmental regulations, including montreal protocol, framework convention on climate change, washington convention, convention on biological diversity, ramsar convention on wetlands, stockholm convention and rotterdam convention; (8) society \& economy, including population \& industry, transportation, energy use, economic benefits, public facilities \& community development and public opinion \& social acceptance. Table V summarizes the use of the three LCIA methods, Eco-indicator 99, IMPACT $2002+$ and ReCiPe, to assess evaluation items in the SEA.

Some items are not evaluated by the three LCIA methods such as waste, noise, non-ionizing radiation, ecological landscape \& habitat, risk of chemical substances, landscape, distribution of water use, water crowding effect, water resource, basel convention, washington convention, convention on biological diversity, ramsar convention on wetlands, stockholm convention, rotterdam convention, population \& industry, transportation, economic benefits, public facilities \& community development and public opinion \& social acceptance.

The results for the use of Eco-indicator 99, IMPACT $2002+$ and ReCiPe to assess case study (2) are shown in Table VI. In Table IV, The results evaluated by Eco-indicator 99 show the most negative impact on human health is 'Respiratory inorganics', the most negative impact on ecosystem quality is 'acidification/ eutrophication', and the most negative impact on resource is 'Fossil fuels'. The results evaluated by IMPACT 2002+ show the most negative impact on human health is 'Respiratory inorganics', the most negative impact on ecosystem quality is 'Terrestrial acid/nutri', and the most negative impact on resource is 'Non-renewable energy'. The results evaluated by ReCiPe 
show the most negative impact on human health is 'Human toxicity', and the most negative impact on ecosystem quality is 'Terrestrial acidification'. Compared to each other, the magnitude of impact evaluated by the three LCIA methods differs up to three orders for human health, eight orders for ecosystem quality and one order for resource. For example, as for A the impact of human health is $8.575 \mathrm{E}+01$ DALY by IMPACT $2002+$, but it raises up to $1.404 \mathrm{E}+04$ DALY by ReCiPe. Meanwhile, as for A the impact of ecosystem quality is $-3.139 \mathrm{E}+00 \mathrm{PDF}^{*} \mathrm{~m}^{2} \mathrm{yr}$ by $\mathrm{ReCiPe}$, but it turns into $-5.161 \mathrm{E}+08 \mathrm{PDF}^{*} \mathrm{~m}^{2} \mathrm{yr}$ by IMPACT $2002+$. The impact of resource is $-1.331 \mathrm{E}+09 \mathrm{MJ}$ surplus by Eco-indicator 99, but it turns into $-2.042 \mathrm{E}+10 \mathrm{MJ}$ surplus by IMPACT 2002+. The difference among LCIA methods primarily comes from their inconsistent characterization models and it makes users confused because they use different models, geographic conditions, time horizons and receptors etc.

TABLE V: THE USE OF LCIA METHODS FOR SEA EVALUATION ITEMS

SEA Evaluation Item Eco-indicator99 IMPACT 2002+ $\mathrm{ReCiPe}$

(A) Assimilative capacity

of the environment

- Air

Climate change

Global warming

Climate change

Ozone layer

Ozone layer

Ozone depletion

depletion

Photochemical

oxidant

formation

Particulate matter

- Water

Acidification/

Eutrophication

Aquatic acidification

formation

Aquatic

Freshwate

eutrophication eutrophication

- Soil

Acidification/

Eutrophication

- Waste

- Non-ionizing radiation

(B) Natural ecology and Ecotoxicity

landscape

- Terrestrial ecosystems

Ecotoxicity

- Aquatic ecosystems

- Ecological landscape

\& habitat

(C) Human Health and Carcinogens

Safety

- Toxic or harmful

substances

$\begin{array}{ll}\text { Respiratory } & \begin{array}{l}\text { Rerganics } \\ \text { Respiratory } \\ \text { inorganics }\end{array} \\ \text { radiation } & \begin{array}{c}\text { Radiation } \\ \text { - Risk of chemical } \\ \text { substances }\end{array} \\ \text { (D) Land resource } & \text { Land use } \\ \text { - Characteristics of land } \\ \text { resources } \\ \text { - Mineral resource \& } \quad \text { Minerals } \\ \text { debris } \\ \text { - Land use } \\ \text { - Landscape } \\ \text { (E) Water resource } \\ \text { 9) Distribution of water } \\ \text { use } \\ \text { 10) Water crowding effect } \\ \text { 11) Water resource } \\ \text { (F) Cultural assets } \\ \text { (G) International } \\ \text { environmental regulations } \\ \text { - Montreal Protocol } \quad \text { Climate change }\end{array}$

Ozone layer

depletion

Global warming
- Framework

Convention on Climate

Change

- Basel Convention

- Washington

Convention

- Convention on

Biological Diversity

- Ramsar Convention on

Wetlands

- Stockholm

Convention

- Rotterdam Convention

(H) Society \& Economy

- Population \& Industry

- Transportation

- Energy use

Fossil fuels

Non-renewable energy

- Economic benefits

- Public facilities \&

community development

- Public opinion \&

social acceptance

\section{DISCUSSION AND CONCLUSION}

TABLE VI: THE RESUlTS FOR THE USE OF LCIA TOOLS FOR CASE STUDY (2)

\begin{tabular}{|c|c|c|c|c|}
\hline \multicolumn{5}{|c|}{ Eco-indicator 99} \\
\hline Impact Category & Unit & $\mathrm{Z}$ & $\mathrm{A}$ & B \\
\hline Carcinogens & DALY & $5.192 \mathrm{E}+00$ & $-3.504 \mathrm{E}+02$ & $2.845 \mathrm{E}+01$ \\
\hline Respiratory organics & DALY & $-7.221 \mathrm{E}-02$ & $5.937 \mathrm{E}-01$ & $8.646 \mathrm{E}-01$ \\
\hline Respiratory inorganics & DALY & $6.606 \mathrm{E}+02$ & $1.668 \mathrm{E}+02$ & $6.427 \mathrm{E}+02$ \\
\hline Climate change & DALY & $-4.950 \mathrm{E}+01$ & $-6.602 \mathrm{E}+01$ & $-1.425 \mathrm{E}+01$ \\
\hline Radiation & DALY & $-7.322 \mathrm{E}-02$ & $-1.879 \mathrm{E}+00$ & $-6.885 \mathrm{E}-02$ \\
\hline Ozone layer & DALY & $-2.943 \mathrm{E}-03$ & $-6.816 \mathrm{E}-02$ & $-2.726 \mathrm{E}-03$ \\
\hline Ecotoxicity & $\mathrm{PDF}^{*} \mathrm{~m}^{2} \mathrm{yr}$ & $1.902 \mathrm{E}+06$ & $-8.061 \mathrm{E}+06$ & $2.927 \mathrm{E}+06$ \\
\hline $\begin{array}{l}\text { Acidification/ } \\
\text { Eutrophication }\end{array}$ & $\mathrm{PDF}^{*} \mathrm{~m}^{2} \mathrm{yr}$ & $3.685 \mathrm{E}+07$ & $9.063 \mathrm{E}+06$ & $3.802 \mathrm{E}+07$ \\
\hline Land use & $\mathrm{PDF}^{*} \mathrm{~m}^{2} \mathrm{yr}$ & $0.000 \mathrm{E}+00$ & $-9.779 \mathrm{E}+06$ & $0.000 \mathrm{E}+00$ \\
\hline Minerals & MJ surplus & $-2.318 \mathrm{E}+05$ & $-9.950 \mathrm{E}+05$ & $-2.179 \mathrm{E}+05$ \\
\hline Fossil fuels & MJ surplus & $-4.512 \mathrm{E}+08$ & $-1.330 \mathrm{E}+09$ & 8.387E+08 \\
\hline Human Health & DALY & $6.161 \mathrm{E}+02$ & $-2.510 \mathrm{E}+02$ & $6.577 \mathrm{E}+02$ \\
\hline Ecosystem Quality & $\mathrm{PDF}^{*} \mathrm{~m}^{2} \mathrm{yr}$ & $3.876 \mathrm{E}+07$ & $-8.777 \mathrm{E}+06$ & $4.094 \mathrm{E}+07$ \\
\hline Resources & MJ surplus & $-4.514 \mathrm{E}+08$ & $-1.331 \mathrm{E}+09$ & $8.385 \mathrm{E}+08$ \\
\hline \multicolumn{5}{|c|}{ IMPACT 2002+ } \\
\hline Impact Category & Unit & $\mathrm{Z}$ & A & B \\
\hline Carcinogens & DALY & $-2.594 \mathrm{E}-01$ & $-1.551 \mathrm{E}+01$ & $3.909 \mathrm{E}-01$ \\
\hline Non-carcinogens & DALY & $3.108 \mathrm{E}+00$ & $-2.925 \mathrm{E}+01$ & $2.135 \mathrm{E}+02$ \\
\hline Respiratory inorganics & DALY & $1.094 \mathrm{E}+03$ & $1.643 \mathrm{E}+02$ & $6.456 \mathrm{E}+02$ \\
\hline Ionizing radiation & DALY & $-7.438 \mathrm{E}-02$ & $-1.879 \mathrm{E}+00$ & $-6.995 \mathrm{E}-02$ \\
\hline Ozone layer depletion & DALY & $-3.118 \mathrm{E}-03$ & $-6.915 \mathrm{E}-02$ & $-2.885 \mathrm{E}-03$ \\
\hline Respiratory organics & DALY & $-7.221 \mathrm{E}-02$ & $5.929 \mathrm{E}-01$ & $8.638 \mathrm{E}-01$ \\
\hline Aquatic ecotoxicity & $\mathrm{PDF}^{*} \mathrm{~m}^{2} * \mathrm{yr}$ & $1.458 \mathrm{E}+05$ & $-5.026 \mathrm{E}+08$ & $2.569 \mathrm{E}+07$ \\
\hline Terrestrial ecotoxicity & $\mathrm{PDF}^{*} \mathrm{~m}^{2} \mathrm{yr}$ & $-4.938 \mathrm{E}+05$ & $-1.273 \mathrm{E}+07$ & $-4.922 \mathrm{E}+05$ \\
\hline Terrestrial acid/nutri & $\mathrm{PDF}^{*} \mathrm{~m}^{2} * \mathrm{yr}$ & $3.682 \mathrm{E}+07$ & $9.055 \mathrm{E}+06$ & $3.798 \mathrm{E}+07$ \\
\hline Land occupation & $\mathrm{PDF}^{*} \mathrm{~m}^{2} * \mathrm{yr}$ & $0.000 \mathrm{E}+00$ & $-9.779 \mathrm{E}+06$ & $0.000 \mathrm{E}+00$ \\
\hline Aquatic acidification & $\mathrm{PDF}^{*} \mathrm{~m}^{2} * \mathrm{yr}$ & & & \\
\hline Aquatic eutrophication & $\mathrm{PDF}^{*} \mathrm{~m}^{2} * \mathrm{yr}$ & & & \\
\hline Global warming & $\mathrm{kg} \mathrm{CO}_{2}$ eq & $-2.134 \mathrm{E}+08$ & $-1.546 \mathrm{E}+08$ & $-6.672 \mathrm{E}+07$ \\
\hline Non-renewable energy & MJ primary & $-5.769 \mathrm{E}+09$ & $-2.042 \mathrm{E}+10$ & $1.076 \mathrm{E}+10$ \\
\hline Mineral extraction & MJ primary & $-2.318 \mathrm{E}+05$ & $-9.950 \mathrm{E}+05$ & $-2.179 \mathrm{E}+05$ \\
\hline Human Health & DALY & $1.052 \mathrm{E}+03$ & $8.575 \mathrm{E}+01$ & $8.463 \mathrm{E}+02$ \\
\hline Ecosystem Quality & $\mathrm{PDF} * \mathrm{~m} 2 \mathrm{yr}$ & $3.647 \mathrm{E}+07$ & $-5.161 \mathrm{E}+08$ & $6.318 \mathrm{E}+07$ \\
\hline Resources & MJ surplus & $-5.769 \mathrm{E}+09$ & $-2.042 \mathrm{E}+10$ & $1.076 \mathrm{E}+10$ \\
\hline \multicolumn{5}{|c|}{$\mathrm{ReCiPe}$} \\
\hline Impact Category & Unit & $\mathrm{Z}$ & A & B \\
\hline $\begin{array}{l}\text { Climate change Human } \\
\text { Health }\end{array}$ & DALY & $-7.517 \mathrm{E}+02$ & $-5.896 \mathrm{E}+02$ & $-2.575 \mathrm{E}+02$ \\
\hline Ozone depletion & DALY & $-4.945 \mathrm{E}-03$ & $-1.626 \mathrm{E}-01$ & $-4.574 \mathrm{E}-03$ \\
\hline Human toxicity & DALY & $3.436 \mathrm{E}+02$ & $1.452 \mathrm{E}+04$ & $6.755 \mathrm{E}+04$ \\
\hline $\begin{array}{l}\text { Photochemical oxidant } \\
\text { formation }\end{array}$ & DALY & 2.391E-01 & $8.751 \mathrm{E}-02$ & $2.872 \mathrm{E}-01$ \\
\hline $\begin{array}{l}\text { Particulate matter } \\
\text { formation }\end{array}$ & DALY & $4.748 \mathrm{E}+02$ & $1.154 \mathrm{E}+02$ & $4.285 \mathrm{E}+02$ \\
\hline
\end{tabular}




\begin{tabular}{|c|c|c|c|c|}
\hline \multirow{2}{*}{$\begin{array}{l}\text { Ionising radiation } \\
\text { Climate change } \\
\text { Ecosystems }\end{array}$} & DALY & $-5.926 \mathrm{E}-02$ & $-1.478 \mathrm{E}+00$ & $-5.573 \mathrm{E}-02$ \\
\hline & species.yr & $-4.004 \mathrm{E}+00$ & $-3.138 \mathrm{E}+00$ & $-1.372 \mathrm{E}+00$ \\
\hline Terrestrial acidification & species.yr & $1.004 \mathrm{E}-01$ & $2.784 \mathrm{E}-02$ & $8.359 \mathrm{E}-02$ \\
\hline Freshwater eutrophication & species.yr & $-7.506 \mathrm{E}-05$ & $-2.507 \mathrm{E}-03$ & $-7.059 \mathrm{E}-05$ \\
\hline Terrestrial ecotoxicity & species.yr & $-2.864 \mathrm{E}-03$ & $-2.441 \mathrm{E}-02$ & $-1.356 \mathrm{E}-03$ \\
\hline Freshwater ecotoxicity & species.yr & $5.396 \mathrm{E}-07$ & $-3.816 \mathrm{E}-04$ & $1.017 \mathrm{E}-03$ \\
\hline Marine ecotoxicity & species.yr & $5.518 \mathrm{E}-05$ & $-8.725 \mathrm{E}-04$ & $1.794 \mathrm{E}-02$ \\
\hline $\begin{array}{l}\text { Agricultural land } \\
\text { occupation }\end{array}$ & species.yr & $0.000 \mathrm{E}+00$ & $0.000 \mathrm{E}+00$ & $0.000 \mathrm{E}+00$ \\
\hline Urban land occupation & species.yr & $0.000 \mathrm{E}+00$ & $0.000 \mathrm{E}+00$ & $0.000 \mathrm{E}+00$ \\
\hline $\begin{array}{l}\text { Natural land } \\
\text { transformation }\end{array}$ & species.yr & $0.000 \mathrm{E}+00$ & $0.000 \mathrm{E}+00$ & $0.000 \mathrm{E}+00$ \\
\hline Metal depletion & $\$$ & $-4.596 \mathrm{E}+04$ & $-5.210 \mathrm{E}+05$ & $-4.323 \mathrm{E}+04$ \\
\hline Fossil depletion & $\$$ & $-2.163 \mathrm{E}+09$ & $-7.831 \mathrm{E}+09$ & 4.139E+09 \\
\hline Human Health & DALY & $6.682 \mathrm{E}+01$ & $1.404 \mathrm{E}+04$ & $6.772 \mathrm{E}+04$ \\
\hline Ecosystem Quality & species.yr & $-3.907 \mathrm{E}+00$ & $-3.139 \mathrm{E}+00$ & $-1.271 \mathrm{E}+00$ \\
\hline Resources & $\$$ & $-2.163 \mathrm{E}+09$ & $-7.831 \mathrm{E}+09$ & 4.139E+09 \\
\hline
\end{tabular}

This study demonstrates the use of LCIA to assess impact in an EIA and a SEA. However, inconsistency in the results for impact using Eco-indicator 99, IMPACT 2002+ and
ReCiPe arise partially because they use different models, geographic conditions, time horizons and receptors.

For case study (1), Table IV shows that the rates of increase in both the quality of the ecosystem and human health are less than $23.9 \%$ if Eco-indicator 99 and $\mathrm{ReCiPe}$ are used, but these values increase to more than $48.3 \%$, if IMPACT $2002+$ is used. The rate of increase in the quality of the ecosystem is greater than that for human health, if Eco-indicator 99 and IMPACT 2002+ are used, but the rate is less if ReCiPe is used.

For case study (2), Table VI shows that the impact of alternative (A) is the least, if Eco-indicator 99 and IMPACT $2002+$ are used, but the least impact falls to the value for the zero alternative, if ReCiPe is used. It should be noted that the negative numbers in Table VI that show a positive impact, are for electricity and coal produced by alternative means. The impact of emissions, consumed diesel, consumed electricity, produced electricity and produced coal are detailed in Table VII.

\begin{tabular}{|c|c|c|c|c|c|c|c|}
\hline Impact Category & Unit & Total & Emissions & $\begin{array}{c}\text { Consumed } \\
\text { Diesel }\end{array}$ & $\begin{array}{l}\text { Consumed } \\
\text { Electricity }\end{array}$ & $\begin{array}{l}\text { Produced } \\
\text { Electricity }\end{array}$ & $\begin{array}{c}\text { Produced } \\
\text { Coal }\end{array}$ \\
\hline Carcinogens & DALY & $-3.504 \mathrm{E}+02$ & $4.235 \mathrm{E}+00$ & $2.675 \mathrm{E}+01$ & $2.512 \mathrm{E}-01$ & $-1.546 \mathrm{E}+00$ & $-3.801 \mathrm{E}+02$ \\
\hline Respiratory organics & DALY & $5.937 \mathrm{E}-01$ & $0.000 \mathrm{E}+00$ & $9.668 \mathrm{E}-01$ & $1.151 \mathrm{E}-02$ & $-7.084 \mathrm{E}-02$ & $-3.138 \mathrm{E}-01$ \\
\hline Respiratory inorganics & DALY & $1.668 \mathrm{E}+02$ & $1.409 \mathrm{E}+02$ & $1.908 \mathrm{E}+02$ & $1.488 \mathrm{E}+01$ & $-9.159 \mathrm{E}+01$ & $-8.825 \mathrm{E}+01$ \\
\hline Climate change & DALY & $-6.602 \mathrm{E}+01$ & $6.364 \mathrm{E}+01$ & $3.418 \mathrm{E}+01$ & $1.871 \mathrm{E}+01$ & $-1.152 \mathrm{E}+02$ & $-6.735 \mathrm{E}+01$ \\
\hline Ozone layer & DALY & $-6.816 \mathrm{E}-02$ & $0.000 \mathrm{E}+00$ & $4.346 \mathrm{E}-05$ & $4.352 \mathrm{E}-04$ & $-2.679 \mathrm{E}-03$ & $-6.596 \mathrm{E}-02$ \\
\hline Ecotoxicity & $\mathrm{PDF}^{*} \mathrm{~m}^{2} \mathrm{yr}$ & $-8.061 \mathrm{E}+06$ & $1.145 \mathrm{E}+06$ & $9.537 \mathrm{E}+05$ & $1.222 \mathrm{E}+05$ & $-7.523 \mathrm{E}+05$ & $-9.530 \mathrm{E}+06$ \\
\hline $\begin{array}{l}\text { Acidification/ } \\
\text { Eutrophication }\end{array}$ & $\mathrm{PDF}^{*} \mathrm{~m}^{2} \mathrm{yr}$ & $9.063 \mathrm{E}+06$ & $7.557 \mathrm{E}+06$ & $7.236 \mathrm{E}+06$ & $5.154 \mathrm{E}+05$ & $-3.173 E+06$ & $-3.072 \mathrm{E}+06$ \\
\hline Land use & $\mathrm{PDF}^{*} \mathrm{~m}^{2} \mathrm{yr}$ & $-9.779 E+06$ & $0.000 \mathrm{E}+00$ & $0.000 \mathrm{E}+00$ & $0.000 \mathrm{E}+00$ & $0.000 \mathrm{E}+00$ & $-9.779 \mathrm{E}+06$ \\
\hline Minerals & MJ surplus & $-9.950 \mathrm{E}+05$ & $0.000 \mathrm{E}+00$ & $0.000 \mathrm{E}+00$ & $3.427 \mathrm{E}+04$ & $-2.110 \mathrm{E}+05$ & $-8.183 E+05$ \\
\hline Human Health & DALY & $-2.510 \mathrm{E}+02$ & $2.088 \mathrm{E}+02$ & $2.527 \mathrm{E}+02$ & $3.386 \mathrm{E}+01$ & $-2.085 \mathrm{E}+02$ & $-5.379 \mathrm{E}+02$ \\
\hline Ecosystem Quality & $\mathrm{PDF}^{*} \mathrm{~m}^{2} \mathrm{yr}$ & $-8.777 \mathrm{E}+06$ & $8.702 \mathrm{E}+06$ & $8.189 \mathrm{E}+06$ & $6.376 \mathrm{E}+05$ & $-3.926 \mathrm{E}+06$ & $-2.238 \mathrm{E}+07$ \\
\hline Resources & MJ surplus & $-1.331 \mathrm{E}+09$ & $0.000 \mathrm{E}+00$ & $1.309 \mathrm{E}+09$ & $6.786 \mathrm{E}+07$ & $-4.178 \mathrm{E}+08$ & $-2.290 \mathrm{E}+09$ \\
\hline
\end{tabular}

\section{ACKNOWLEDGMENT}

The authors would like to thank the Ministry of Science and Technology of the Republic of China (Taiwan) for financially supporting this research under Contract MOST 103-2221-E-131-001-MY2.

\section{REFERENCES}

[1] K. F.-R. Liu, H.-H. Liang, C.-W. Chen, J.-S. Chen, and Y.-S. Shen, "Combining scientific facts and significance criteria to predict the result of an environmental impact assessment review," Journal of Environmental Informatics, vol. 19, no. 2, pp. 93-107, June 2012.

[2] B. Sadler and R. Verheem, SEA: Status, Challenges and Future Directions, Report 53, Ministry of Housing, Spatial Planning and the Environment, The Hague, Netherlands, 1996.

[3] K. F.-R. Liu, C.-Y. Ko, C. Fan, and C.-W. Chen, "Incorporating the LCIA concept into fuzzy risk assessment as a tool for environmental impact assessment," Stochastic Environmental Research and Risk Assessment, vol. 27, no. 4, pp. 849-866, May 2013.

[4] ISO 14040: Environmental management — Life cycle assessment principles and framework, International Organisation for Standardisation (ISO), Geneve, 2006.

[5] ISO 14044: Environmental management — Life cycle assessment requirements and guidelines, International Organisation for Standardisation (ISO), Geneve, 2006.
[6] M. J. Goedkoop and R. Spriensma, "The eco-indicator 99 - A damage-oriented method for life cycle impact assessment," Methodology Report, Pre' Consultants, B.V. Amersfoort, The Netherlands, 2000.

[7] O. Jolliet, M. Margni, R. Charles, S. Humbert, J. Payet, G. Rebitzer, and R. Rosenbaum, "IMPACT 2002+: A new life cycle impact assessment methodology," The International Journal of Life Cycle Assessment, vol. 8, no. 6, pp. 324-330, 2003.

[8] M. J. Goedkoop, R. Heijungs, M. Huijbregts, A. D. Schryver, J. Struijs, and R. Van Zelm, "ReCiPe 2008, A life cycle impact assessment method which comprises harmonised category indicators at the midpoint and the endpoint level," First Edition Report I: Characterisation, 6 January, 2009.

[9] Institute for Environment and Sustainability, Joint Research Centre of European Commission. (2010b). Framework and requirements for Life Cycle Impact Assessment (LCIA) models and indicators. [Online]. Available:

http://lct.jrc.ec.europa.eu/pdf-directory/ILCD-Handbook-LCIA-Fram ework-requirements-online-12March2010.pdf

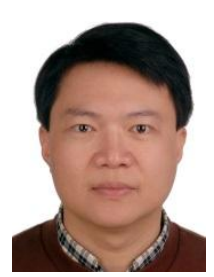

Kevin Liu received the Ph.D. degree in 1998 in civil engineering from National Central University, Taiwan. Currently, he is a professor in the Department of Safety, Health and Environmental Engineering, Ming Chi University of Technology, Taiwan. His research interest is the use of decision analysis and artificial intelligence techniques to environmental management issues. 
Si-Yu Chiu received the master degree in 2011 in the Department of Safety, Health and Environmental Engineering, Ming Chi University of Technology, Taiwan. She currently works for an environmental management company. Her research interest is the uncertainty analysis in LCA.

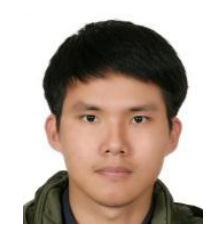

Po-Chung Yeh received the master degree in 2013 in the Department of Safety, Health and Environmental Engineering, Ming Chi University of Technology, Taiwan. He currently works for an environmental engineering company. He research interest is the application of GIS and LIA to SEA.

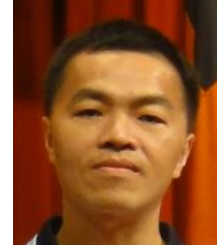

Jong-Yih Kuo received the Ph.D. degree in 1998, computer science and information engineering from National Central University, Taiwan. Currently, he is an associate professor in the Department of Computer Science and Information Engineering, National Taipei University of Technology, Taiwan. His research interest is agent-base software engineering, intelligent agent system, agent system development, web-base application, machine learning and fuzzy theory. 